\section{THE STRUCTURE OF THE UNIVERSE*}

\author{
By SIR JAMES JEANS, O.M., F.R.S.
}

\section{$\mathrm{T}$} HE earliest astronomy was geocentric, the earth being supposed to be the centre of the whole universe. This view was not based on astronomical evidence, but had its roots in man's self-esteem, in his want of imagination and in the meagreness of his scientific knowledge. It met its end in the arguments of Copernicus and in the observations of Galileo.

It was succeeded by what we may call a heliocentric astronomy, in which the sun was supposed to be at or near the centre of the galactic system, and possibly also of the whole universe. This view did not result from any human frailty; there seemed to be good scientific evidence for it. For a superficial study of the sky shows that those stars that appear brightest to us, and so are presumably nearest to us, are scattered fairly uniformly in the different directions of space, while the Milky Way divides the sky into exactly equal halves, and itself looks about equally bright in all its parts. All this seemed to indicate a disk-shaped system of stars, with the sun lying in the central plane of the disk, and fairly close to its centre. Such a view of the structure of the galaxy appeared to find confirmation in the pioneer researches of the two Herschels, and in the later investigations of Kapteyn, Seares and others.

We know now that it was entirely wrong. It was wrong because these investigators had assumed that space was entirely transparent to light. We know now that the whole galactic system is permeated by a patchy fog of obscuring matter which is not dense enough to affect the light of the nearer stars appreciably, but blots out the more distant stars entirely. This fog makes the greater part of the galactic system invisible to us; if our predecessors thought they were at the centre of the galactic system, it was as a man who is in a forest in a thick fog may think he is at the centre of the forest, although in truth he is only at the centre of the small group of trees he can see through the fog.

We now know that the centre of the galaxy is far removed from the sun, and that the sun, like all the rest of the galaxy, is revolving around this distant centre. Observation shows that the sun's period of rotation is about 250 million years, and that its orbital speed is of the order of $270 \mathrm{~km}$. a second. These purely observational data show that the galactic centre must be at a distance of about 36,000 light-years from the sun.

It used to be thought that our own particular galaxy was far larger than any other in the sky, but it is now clear that all galaxies are very similar in size; and also very similar in mass. Our own galactic system can be weighed by calculating the gravitational force it exerts on the sun to keep this moving in the orbit already described; the requisite mass comes out at about 150,000 million suns. It is also possible to weigh a close pair or cluster of galaxies by calculating the gravitational force they must exert on one another so as to prevent the more rapidly moving members running away into space. The average mass needed usually comes out at about that just mentioned for our own galaxy, most estimates ranging from 95,000 million to 200,000 million suns. On the evidence at present available, the

\footnotetext{
* Discourse delivered at the Royal Institution on March 26.
}

galaxies seem likely to differ far less one from another than the stars of which they are composed, and we are led to picture the astronomical universe as consisting of a number of similar units-our own galaxy and the other galaxies - rather like the molecules of a gas.

On pointing a telescope in different directions in space, we see fields of stars which differ greatly from one another-a consequence of the finite size and definite structure of the galaxy. No comparable variations are to be found in the fields of galaxies seen in different directions in space. Clusters may be seen here and there, and also bare patches in places, but broadly speaking the galaxies seem to be scattered fairly evenly through space, the average distance of neighbours being something more than a million light-years. We do not know whether this uniformity of distribution persists through the whole of space or not. For the galaxies that we can see may perhaps form only a small part of some grander system, built on such a scale that no appreciable difference of structure occurs within the distances accessible to our telescopes.

If, however, the distribution is uniform throughout the whole of space, then space must be finite; otherwise it would contain an infinite amount of matter, and the gravitational force from this would be infinite, which is contrary to the fact. This alterna. tive, then, brings us to consideration of the type of universe which Einstein contemplated in his original relativity theory. Space is curved with a positive curvature-like the surface of the earth-and is filled with matter of which the density, when averaged through a sufficiently large volume of space, is everywhere the same. On this theory the size of space is determined uniquely by this average density of matter, much as the size of an expanded balloon depends on the density, and so on the pressure, of the gas inside it. From the data already mentioned, we can deduce that the average density of matter in space must be of the order of $10^{-28} \mathrm{gm}$. per c.c., or about one atom to the cubic yard. With this density the radius of space would be about 3,300 million light-years-at least if the whole structure is at rest in a configuration of equilibrium. Thus the whole range of our biggest telescopes would be only a minute fraction of the size of space.

This seemed to provide a possible and consistent scheme until Friedmann and Lemaître showed that such a universe could not stand at rest in equilibrium. It would be unstable in the sense that space itself would have to start either expanding or contracting. Some time after this, Hubble and Humason found displacements in the spectra of distant nebulæ which, if interpreted in the simplest way as 'Doppler effects', showed that the distant nebulæ were all receding from us, and this seemed to suggest that space might actually be expanding just as Friedmann and Lemaitre had predicted. Observation showed that the rate of expansion would be the same everywhere and such that, if it were maintained at its present value, the linear dimensions of space would be doubled in about 1,800 million years.

This in turn suggested that the universe might have started as an Einstein universe of the kind already described, and that the inherent instability of such a configuration had caused it to expand to its present dimensions. But the theory of relativity could not deduce the present dimensions, either from the present density of matter or otherwise, Einstein's relation between size and density referring only to a universe at rest in equilibrium. 
Eddington has claimed to solve the problem by quite different methods. In brief, he believes that the total number of protons in the universe must be $136 \times 2^{256}$, there being also an equal number of electrons; he has produced arguments to show that the universe cannot, from the nature of things, be anything other than this.

Knowing the number of particles in the universe, and the mass of each, it is easy to calculate the total mass of the universe, and hence the dimensions, since we already know the average density to be about $10^{-28}$; the radius of curvature comes out at some 2,000 or 3,000 millions of light-years. But if the universe started as an Einstein universe in equilibrium, then the total mass it contained-the known total mass of all Eddington's particles-would fix its curvature definitely and precisely. Eddington calculates that the radius of curvature would then be 1,068 millions of light-years. Thus the expansion of the universe up to the present can only have increased its dimensions some two- or three-fold, a process which would occupy only a few thousands of millions of years. This agrees well enough with what we know as to the age of the earth, for it seems probable that the earth came into existence between 2,000 and 3,000 millions of years ago, the sun then being at the very beginning of its existence as a star. It also agrees with what we know as to the ages of the stars in general; if present conjectures as to the mechanism of stellar radiation are right, the stars can scarcely have contained available energy to provide radiation for more than a very few thousands of millions of years.

The problem has been attacked on different lines by Milne, Dirac and others. After the geocentric and the heliocentric universes had been banished from astronomy, the apparent recessions of the nebulæ seemed to suggest a galacto-centric universe, with our own galaxy as the centre from which all others were moving radially away. Milne based his theory on what he described as the 'cosmological principle' - the universe is not in any way centred in our galaxy ; this occupies no specially favoured position, so that the picture which an inhabitant of our galaxy draws of the large-scale features of the universe would be equally valid for any other galaxy.

With the help of this principle, Milne draws a picture which seems at first sight to describe something totally different from the expanding universe of Eriedmann and Lemaître. But Kermack and McCrea claim to have shown that the two pictures differ only in the way in which two maps of the same country differ when they are drawn on different projections. If we compare the relativity picture to a spherical projection, then Milne's picture may be compared to a Mercator projection; and MeVittio has recently advanced the claim that Milne's results do not really depend on the cosmological principle at all, but can all be deduced from assumptions which Milne has unwittingly introduced under an erroneous impression that they are axiomatic. This matter is still under discussion.

Another line of investigation was opened by Dirac, possibly under the influence of a remark which Eddington had made so far back as 1923. Physics provides a natural unit of force, namely, the attraction between the nucleus and the electron in the hydrogen atom. Astronomy provides another natural unit of force-the force with which the same two particles attract one another gravitationally. This latter unit is very small in comparison with the physical unit, the two standing in the ratio of $2 \cdot 3 \times 10^{38}$ to 1 . This ratio is of course a pure number, and so is independent of the particular units we use for our measurements. We have already mentioned another large number-the number of protons in the universe, which Eddington claims must be $1.54 \times 10^{79}$. The square root of this number is $3.9 \times 10^{39}$, and so is very close to the ratio of the electric and gravitational forces just mentioned. Eddington and Dirac have both suggested that the agreement is too good to be the result of a mere coincidence; they prefer to think that it must result from some fundamental property of the universe.

Again, physics provides a natural unit of time. It can be expressed in a variety of forms, the simplest being that it is the time light takes to travel across the diameter of an electron, about $1.25 \times 10^{-23} \mathrm{sec}$. Astronomy also provides a natural unit of time; it is the fictitious time that the various galaxies would take to reach their present positions if they were all to start simultaneously from a point and travel uniformly at their present speeds of motion. This time is the same for all galaxies, being about 1,800 millions of years. Again, the ratio of these two units is a very large number; this time it is $4.5 \times 10^{30}$, which is very near to the large numbers already mentioned. Again we must assume that the agreement is not a mere accident, but must represent something in the order of Nature.

We do not know what is the explanation of these apparent coincidences, but they seem to conceal something not yet fully explained, which when fully understood may prove to be of outstanding importance. It is true that Eddington's researches provide an explanation, but only in a rather special and very recondite form; it looks as though this explanation may be only a special case of something simpler and far wider. The fundamental constants of Nature seem to be connected by some new and hitherto unsuspected relations.

Some investigators have gone further than this, and traverse, as it seems to me, very dangerous ground. The age of the universe, expressed in terms of the natural physical unit of time, proves once again to be of the order of $10^{38}$, and the suggestion is made that this also can be no mere accident, but must express something in the fixed order of Nature. The weakness in this argument is, as it seems to me, that the basic fact on which it rests can be put in the simpler form that the age of the universe is some one, two or three times the time in which space doubles its dimensions; and that when it is so expressed, it is difficult to find any 'coincidence', either real or apparent. The supposed coincidence is seen to be merely a simple transformation of one which has already been dealt with, and we must not, so to speak, try to cash in on the same coincidence twice over.

Let us, however, waive the objection and accept the suggestion made. We have then to suppose that all the numbers of the order of $10^{39}$, including the age of the universe, owe their approximate equality to something in the fixed order of Nature ; when the universe attains to ten times its present age, the $10^{39}$ which measures its age will have increased to $10^{40}$, and all the other big numbers will have done likewise. Thus, the ratio of electrical to gravitational attraction will be ten times what it now is, and so on, while the number of particles in the universe will have increased a hundred-fold, so that creation must still be in progress. In brief, some or all of the quantities that we used to regard as unalterable 
constants of Nature lose their quality of constancy, and must change continually with the time. Milne was led to the same conclusion by a very different road; he reached it from a study of his cosmological principle. By whatever road we arrive, we come into a fantastic new world.

We may avoid the need for a continual creation of matter by supposing that the natural physical unit of time changes pari passu with the age of the universe. Then the measure of the age of the universe stays always the same, as does also the number of particles in the universe. But we now find that either the mass or the charge of an electron must continually change.

All this seems strange to old-fashioned physics, but it simplifies some things and removes some difficulties. When, for example, we study the spectrum of a nebula at 250 million light-years distance, we are in effect watching the emission of light from the atoms of 250 million years ago. We find that the spectral lines all show a displacement to the red, its amount being strictly proportional to the age of the light. The simplest interpretation of what we see is that the atoms of those earlier days were not the same as the atoms of to-day. Atoms seem to have given out radiation of longer wave-length than they do now, and so were apparently larger-or perhaps their electrons moved more slowly in their orbits and so took longer to complete their revolutions, possibly because the electric attraction on them was less intense. There are many possibilities, each with its merits, but also with many demerits.

Whatever the final solution of this vast problem may prove to be, it is already clear that there is no solution on the lines of the kind of dynamics that we learned at school. The mechanical interpretation of the universe fails as completely in the large-scale world of astronomy as it has already failed in the small-scale world of atomic physics. The quantum theory has replaced mechanics in the physical world; we still do not know what is destined to replace it in the world of astronomy.

\section{CULTIVATION OF THE DOUGLAS FIR IN GREAT BRITAIN}

\section{BY ALEXANDER L. HOWARD}

$\mathrm{T}$ $\mathrm{HE}$ question of reconstruction after the War is uppermost in many thinking minds to-day, and reafforestation should be given an important place on the programme.

Until the year 1914 a period of nearly a hundred years elapsed, during which time in Great Britain only desultory planting of little importance was carried out. The War of 1914-18 came, bringing with it a merciless but unavoidable devastation of our forests, and subsequent efforts proved wholly inadequate to restore our position. If the situation was serious then, it is desperate now, as the huge demand for timber which has arisen since the outbreak of war in 1939 has dealt another crippling blow to the woodlands of Britain. It is easy to see how this grave situation has come about. In the years 1937 and 1938 (1939 was not a complete year) we imported from the U.S.S.R., Finland, Sweden, Poland, Norway and elsewhere (including Canada and America) the following :
1937
Softwoods
$11,732,000$ loads
Money value (sterling)
$8,660,000$
$£ 51,574,000$

So as to make the immensity of this problem clearer to those who are less acquainted with the matter :

The quantity imported in 1937 would weigh approximately, of our class of timber

The transport of which would employ

(each with an average capacity of 6 tons,

but which in practice generally would average, for timber, only half the quantity
named.)

If the full 6 tons were carried, this would

require of the average carrying capacity of

timber over the railways of Britain 133,400 goods trains.

These immense shipments were suddenly and completely cut off, and we were obliged to rely on those supplies which could still be obtained from Canada, the United States, and our own home-grown forests. The largest contribution, which has consisted of Douglas fir, has so far been provided by Canada, and perhaps admittedly or unknowingly by the United States.

The Douglas fir, which flourishes in the north-west coast of North America, is remarkable for its height and size, its rapid growth, and the amazing extent of its usefulness.

According to Pliny, when Xerxes first saw the plane tree (Platanus orientalis) in southern Europe, he halted his entire army of 170,000 soldiers to admire the "pulchritude and procerity" of one single tree. Well might he have done so when he beheld for the first time the majestic height and wonderfully straight bole of the Douglas fir. Although, however, in places other than its natural habitat its lower branches grow in a graceful sweep, for sheer beauty it cannot be considered a competitor with the plane.

Gibson, speaking of the Douglas fir, wrote: "The largest are 300 feet high, occasionally more, and from 8 to 10 feet in diameter. The average among the Rocky Mountains is from 80 to 100 feet high and 2 to 4 feet in diameter. The amount of timber yielded by one tree may be realised from the experience of Dr. Watney (of 'Buckholt', Pangbourne), who was present at the felling of one in Washington Territory, U.S.A. The height of the trunk was 250 feet and that to the lowest bough was 157 feet. The following were the diameters at different heights above the ground :

" 83 inches at 7 feet, 65 inches at 37 feet, 52 inches at 107 feet, and 32 at 191 feet.

"The trunk was sawn off at a height of 7 feet above the ground (where it showed 420 annual rings), and 184 feet of its length yielded 21,503 feet converted, equalling 1,958 feet cube. It took nine railway trucks to convey the timber from London to Pangbourne. The timber contained practically no sap, very few shakes, but some of the planks contained dead knots. Large sections of the trunks (exceeding 7 feet in diameter) are familiar in England to those who visit Kew Gardens, and the Natural History Museum, South Kensington. The Douglas fir flagstaff formerly at Kew Gardens, which was presented by the Government of British Columbia in 1861, was well known. It was 159 feet in length, and measured 1 foot 8 inches in diameter at the base, and 5 inches in diameter at the small end. This is now surpassed by the flagstaff which was erected in 1919 and, like the former one, it was presented by the Government of British Columbia."

The present flagstaff at Kew towers to a height of $214 \mathrm{ft}$., half as high again as the Nelson Monument 